\title{
Agravos à saúde mental de pessoas idosas frente a COVID-19
}

\author{
Worsening mental health of elderly people in front of COVID-19 \\ Empeoramiento de la salud mental de las personas mayores frente al COVID-19
}

Recebido: 08/06/2021 | Revisado: 15/06/2021 | Aceito: 17/06/2021 | Publicado: 30/06/2021

\author{
Analise Lasari Peres Canali \\ ORCID: https://orcid.org/0000-0001-6302-9300 \\ Universidade de Passo Fundo, Brasil \\ E-mail: analasari@gmail.com \\ Silvana Alba Scortegagna \\ ORCID: http://orcid.org/0000-0002-5100-6459 \\ Universidade de Passo Fundo, Brasil \\ E-mail: silvanalba@upf.br
}

\begin{abstract}
Resumo
O novo coronavírus responsável pela doença infecciosa denominada COVID-19, atinge potencialmente grupos de risco com comorbidades, como as pessoas idosas. O presente estudo teve como objetivo verificar o impacto causado pela pandemia do COVID-19 na saúde mental de adultos idosos. Metodologia: A revisão sistemática seguiu as recomendações do Preferred Reporting Items for Systematic Reviews and Meta-Analyses PRISMA (Moher et al., 2009). A busca de artigos deu-se nas bases de dados Pepsic, Pubmed, Science Direct, Gerontology e Scielo. A seleção de dados buscou sistematizar informações sobre objetivos dos estudos, metodologia utilizada, população estudada e os resultados alcançados. Resultados: Foram localizados 408 artigos, selecionados 12, sendo nove de origem nacional e três estrangeiros. Os efeitos da pandemia foram relacionados ao isolamento e distanciamento social e evidenciados em sintomas de ansiedade, depressão, estresse, alterações no sono e na alimentação, dentre outros. Conclusão: Diante da pandemia COVID-19 e de seus efeitos, prover serviços coletivos transdisciplinares de suporte psicossocial, na modalidade on-line, e atentar para sinais de agravos à saúde mental e condições gerais de saúde de adultos idosos, são medidas de saúde pública prioritárias.
\end{abstract}

Palavras-chave: Cuidado do idoso; Envelhecimento; Saúde mental; Serviços de saúde pública.

\begin{abstract}
The new coronavirus responsible for the infectious disease called COVID-19 potentially affects risk groups with comorbidities, such as the elderly. The present study aimed to verify the impact caused by the COVID-19 pandemic on the mental health of elderly adults. Methodology: The systematic review followed the recommendations of the Preferred Reporting Items for Systematic Reviews and PRISMA Meta-Analyses (Moher et al., 2009). The search for articles was carried out in the Pepsic, Pubmed, Science Direct, Gerontology and Scielo databases. Data selection sought to systematize information on study objectives, methodology used, population studied and results achieved. Results: 408 articles were located, 12 were selected, nine of national origin and three foreign. The effects of the pandemic were related to isolation and social distancing and evidenced in symptoms of anxiety, depression, stress, changes in sleep and eating, among others. Conclusion: In view of the COVID-19 pandemic and its effects, providing transdisciplinary collective psychosocial support services, online, and paying attention to signs of damage to mental health and general health conditions of elderly adults, are public health measures priority.
\end{abstract}

Keywords: Elderly care; Aging; Mental health; Public health services.

\section{Resumen}

El nuevo coronavirus responsable de la enfermedad infecciosa denominada COVID-19 afecta potencialmente a grupos de riesgo con comorbilidades, como los ancianos. El presente estudio tuvo como objetivo verificar el impacto causado por la pandemia COVID-19 en la salud mental de los adultos mayores. Metodología: La revisión sistemática siguió las recomendaciones de los ítems de informes preferidos para revisiones sistemáticas y metaanálisis PRISMA (Moher et al., 2009). La búsqueda de artículos se realizó en las bases de datos Pepsic, Pubmed, Science Direct, Gerontology y Scielo. La selección de datos buscó sistematizar información sobre los objetivos del estudio, la metodología utilizada, la población estudiada y los resultados obtenidos. Resultados: se localizaron 408 artículos, se seleccionaron 12, nueve de origen nacional y tres extranjeros. Los efectos de la pandemia se relacionaron con el aislamiento y el distanciamiento social y se evidenciaron en síntomas de ansiedad, depresión, estrés, cambios en el sueño y la alimentación, entre otros. Conclusión: Ante la pandemia de COVID-19 y sus efectos, la prestación de servicios de apoyo psicosocial colectivo transdisciplinario, en línea, y la atención a los signos de daño a la salud mental y las condiciones generales de salud de los adultos mayores, son medidas de salud pública prioritarias.

Palabras clave: Cuidado de los ancianos; Envejecimiento; Salud mental; Servicios de salud pública. 


\section{Introdução}

Desde o início do ano de 2020, as populações do mundo vivem um período atípico e incerto com o avanço e a disseminação de uma nova doença. De acordo com a Organização Mundial da Saúde (OMS), em 30 de janeiro de 2020, surgia o alerta sobre um surto causado por um novo coronavírus, cuja doença foi denominada Coronavirus Disease 2019 (COVID19). Este alerta gerou uma Emergência de Saúde Pública de importância mundial e a necessidade de atualizar o Regulamento Sanitário Internacional, que tinha sua última versão em 2005. Em 11 de março de 2020, a COVID-19 foi considerada pela OMS como uma pandemia (OPAS, 2020).

A síndrome respiratória gerada pela COVID-19 foi identificada primeiramente em 2019, na cidade chinesa de Wuhan. Esta síndrome que vem acometendo as pessoas, pode apresentar diversos níveis de sintomas, e pode levar à morte. Em quadros mais graves, se fez necessário internações hospitalares e cuidados específicos, como o uso de ventiladores mecânicos para controlar a insuficiência respiratória (Faro et al., 2020). Os quadros de maior gravidade sintomatológica atingem principalmente, grupos mais vulneráveis como as pessoas idosas e profissionais da saúde que se encontram na linha de frente de combate ao vírus.

Passado um ano do surgimento do COVID-19, o mundo contabiliza mais de 2.700.000 óbitos. No Brasil, atualmente o número de óbitos já ultrapassa a casa dos 290.000. De acordo com a análise, até o início de outubro de 2020, foram notificados 210.007 casos e 100.059 óbitos de pessoas de 60 anos ou mais - o que corresponde a 53,1\% do total de casos e 75,2\% dos óbitos -, comprovando a maior gravidade da COVID-19 entre a população idosa (FIOCRUZ, 2020).

Os adultos idosos são mais vulneráveis a enfermidade por fatores que afetam a saúde geral, devido às alterações sofridas pelo sistema imunológico. Ã medida que a pessoa envelhece torna-se mais difícil para o sistema imunológico eliminar as células infectadas e transmitir os sinais de alerta para que sejam acionados os mecanismos de defesa do organismo. Com isso, o vírus tem mais tempo de se espalhar aumentando as chances de que os sintomas sejam agravados, podendo levar a óbito. Outro fator a ser considerado é que muitos adultos idosos apresentam comorbidades como: hipertensão, diabetes, doenças renais, doenças pulmonares, dentre outras, o que aumenta o risco de infecção e complicações clínicas de saúde (OMS, 2020).

A saúde mental de adultos idosos também pode ser afetada pelo isolamento físico e social, o medo de contrair a doença, pode gerar um alto grau de sofrimento psíquico prejudicial, e incidir sobre a emergência de quadros de ansiedade e depressão. Em alguns casos, o desafio está em preservar a autonomia, pois pode haver deterioração muscular causada pela falta de atividade física ou má alimentação. Para tanto, é necessário que se mantenham boas condições de saúde e controle de doenças para o não agravamento e possível hospitalização no enfrentamento de pandemias. Indivíduos com idades acima de 60 anos se enquadram no grupo de risco, mesmo sem ter comorbidades associadas (OMS, 2020).

Nesse contexto maléfico, desde o surgimento dos primeiros casos de COVID-19, para conter o avanço desse inimigo invisível, algumas medidas de prevenção foram adotadas, como por exemplo: o distanciamento físico e social, que é a distância espacial necessária, aproximadamente dois metros das pessoas, quando estiver em locais públicos; evitar lugares com aglomeração de pessoas; a quarentena, que é a medida adotada para diminuir a circulação de pessoas que foram expostas a doença. Como a transmissão do novo coronavírus ocorre por meio do ar ou do contato com partículas contaminadas, outras medidas de prevenção adotadas como lavar com frequência as mãos, usar álcool em gel 70\%, não tocar em mucosas como nariz, boca e olhos sem antes higienizar as mãos, evitar contato físico, usar máscaras, não compartilhar objetos de uso pessoal, entre outros (Ministério da Saúde, 2020). Segundo a Organização Pan-Americana da Saúde (OPAS), é fundamental manter as medidas de proteção, lavar frequentemente as mãos com água e sabão ou passar álcool em gel e cobrir a boca com o antebraço ou lenço quando tossir ou espirrar. 
O isolamento social é fundamental para conter o avanço da pandemia, porém manter pessoas afastadas de outras pessoas e de suas atividades rotineiras, apesar dos benefícios, tende a gerar situações desagradáveis que podem ocasionar impactos na saúde mental. Manter-se pessoas afastadas de amigos e familiares e não saber ao certo até quando esse distanciamento será necessário, pode se tornar um fator iminentemente estressante (Faro et al, 2020), provocar o surgimento e/ou o aumento de sentimentos de ansiedade, insegurança e medo (Hossain et al., 2020).

O medo da contaminação e o isolamento social imposto pelas medidas protetivas para conter o vírus, impulsionou o aparecimento de alterações na saúde mental, principalmente de adultos idosos, deixando-os mais ansiosos e vulneráveis diante das incertezas que afetam seu futuro (Lima et al., 2020). Dentro desta conjuntura, a saúde mental é um elemento fundamental para saúde geral dos indivíduos, bem como da coletividade, visto que as pessoas têm pensamentos, emoções e interagem entre si. Assim sendo, promover, proteger e restaurar a saúde mental é vital não só para as pessoas, mas para toda a sociedade (Who, 2020a).

Para alguns pesquisadores (Brooks et al., 2020; Van Orden, 2020; Whitehead, 2020), dentre outros, a sintomatologia psicológica da ansiedade e quadros depressivos estão interligadas as etapas da doença. Primeiramente pode-se apontar as mudanças sofridas no cotidiano dessas pessoas causando desequilíbrios neurofisiológicos ativados pelo estresse (Wang et al.,2020). Num segundo momento o distanciamento social muda à rotina dos adultos idosos fazendo com que se sintam entediados e desamparados, expressando emoções como ansiedade, irritabilidade (Santos, Brandão \& Araújo, 2020). Ainda de acordo com os autores, a última etapa relaciona-se as sequelas deixadas pela pandemia, a diminuição do poder econômico, as perdas afetivas, mortes e lutos por entes e pessoas queridas e, ainda, traumas em pessoas que precisaram ser internadas.

As pessoas idosas que vivem sozinhas podem sofrer de solidão devido ao maior isolamento social e a interrupção das atividades do grupo. São necessárias ideias criativas para incorporar atividades físicas e sociais seguras. Enquanto pessoas jovens estão recorrendo à tecnologia, por meio de smartphones e computadores, para permanecerem socialmente ativos, os idosos podem ter dificuldades para fazer uso dessas ferramentas e se não possuírem algum familiar ou cuidador que possa auxiliar (Brown et al., 2020). Deve-se ter o cuidado e analisar como ajudar esse grupo de risco, pois auxiliar sobre possíveis novas maneiras de se relacionar com outras pessoas pode funcionar para alguns, mas provavelmente não funcionará para todos. O condicionamento das relações com outras pessoas geralmente é moldado pelas raízes familiares, culturais e geracionais, e muitos adultos idosos que viveram a maior parte de suas vidas em comunidades que ofereciam formas naturais de conexão social, como clubes sociais, organizações comunitárias, igrejas e atividades patrocinadas pelo local de trabalho, podem ter dificuldades em se adaptar a esta nova realidade (Lima, 2020).

Existem muitas barreiras emocionais para mudar os comportamentos sociais que tornam a manutenção de hábitos mais confortável e menos trabalhosa, embora o isolamento e a solidão sejam dolorosos (Van Orden et al.,2020). No intuito de conhecer melhor esta realidade e contribuir para o seu enfrentamento, este estudo buscou verificar o impacto causado pela pandemia do COVID-19 na saúde mental de pessoas idosas.

\section{Metodologia}

Foi realizada uma revisão sistemática a partir das recomendações do Preferred Reporting Items for Systematic Reviews and Meta-Analyses PRISMA (Moher et al., 2009). A revisão sistemática, é uma forma de pesquisa que utiliza como fonte de dados a literatura sobre determinado tema. Esse tipo de investigação disponibiliza um resumo das evidências relacionadas a uma estratégia de intervenção específica, mediante a aplicação de métodos explícitos e sistematizados de busca, apreciação crítica e síntese da informação selecionada. Considerando que a pandemia teve início oficial no começo de 2020 (OMS, 2020), a busca de artigos nacionais e internacionais considerou o período de janeiro a dezembro de 2020 . Como critério 
de elegibilidade a este estudo foram inseridos artigos que abordaram os impactos da pandemia do COVID 19 na saúde mental das pessoas idosas. Excluíram-se os artigos que não se encaixavam no objetivo proposto por este estudo. A busca de artigos deu-se nas bases de dados Pepsic, Pubmed, Science Direct, Gerontology e Scielo, com o uso dos descritores: "saúde mental”, “isolamento social”, “idosos", COVID 19 e "pandemia”. Após a leitura inicial dos títulos e resumos de todos os artigos, foram incluídos aqueles que traziam conteúdos referentes a saúde mental, a pandemia do COVID 19, ao isolamento social e aos idosos. A seleção de dados buscou sistematizar informações sobre objetivos de cada estudo, as características metodológicas, população estudada e os resultados apurados.

Os resultados da busca na literatura científica realizada nas bases de dados nacionais e internacionais totalizaram 408 artigos, sendo excluídos um total de 396 por não contemplar a temática proposta no estudo, a saúde mental da população idosa no enfrentamento a pandemia; alguns por fazerem referência somente ao Covid-19; outros por tratarem da saúde mental de profissionais da saúde e outras classes de pessoas. Dos artigos restantes, 12 abordaram os impactos da pandemia na saúde mental dos idosos. A Figura 1 apresenta o fluxograma da estratégia de busca utilizada neste trabalho. 
Figura 1. Fluxograma demonstrativo da estratégia de busca nas bases de dados.

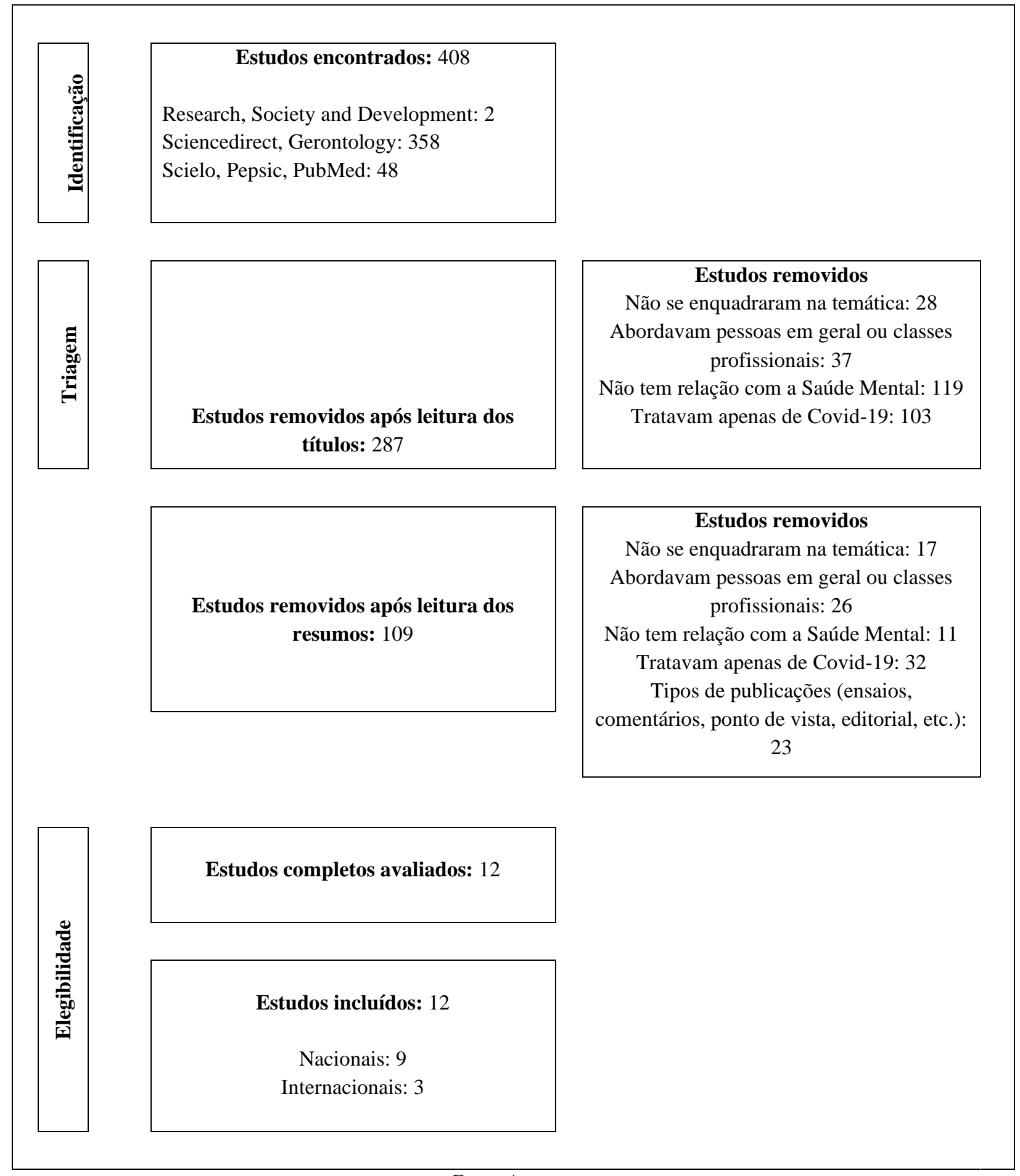

Fonte: Autores.

\section{Resultados}

A verificação e a sistematização dos dados em vários elementos de investigação, separados em aspectos formais e de conteúdo. No Quadro 1 pode-se verificar os artigos publicados contendo autor, pais, população pesquisada, objetivo do estudo, método e principais resultados. 
Quadro 1. Descrição dos estudos incluídos para análise neste estudo.

\begin{tabular}{|c|c|c|c|c|}
\hline Autor/País & População & Objetivo & Método & Principais Resultados \\
\hline $\begin{array}{l}\text { Garcia, Elen } \text { Bauer; } \\
\text { Machado, Laura } \\
(2020) \text { - Brasil }\end{array}$ & $\begin{array}{l}\text { Idosos acima } \\
\text { de } 65 \text { anos }\end{array}$ & $\begin{array}{lr}\text { Compreender } & \text { os } \\
\text { aspectos referente a } \\
\text { saúde mental } \\
\text { emocional } & \text { de } \\
\text { pessoas idosas } & \text { no } \\
\text { contexto } & \text { da } \\
\text { pandemia } & \text { por } \\
\text { COVID-19. } & \\
\text { Refletir sobre como } \\
\text { o cenário } & \text { da } \\
\text { pandemia } & \text { tem } \\
\text { impacto a saúde } \\
\text { emocional } & \text { dos } \\
\text { idosos. } & \end{array}$ & \begin{tabular}{lr}
\multicolumn{2}{c}{ Revisão narrativa de } \\
literatura, & os \\
materiais & foram \\
selecionados & de \\
acordo com & a \\
temática. &
\end{tabular} & $\begin{array}{l}\text { Há particularidades no modo } \\
\text { como a pandemia impacta as } \\
\text { pessoas idosas, considerando } \\
\text { seu nível de saúde anterior a } \\
\text { pandemia, mas de modo geral } \\
\text { foram relacionados sentimentos } \\
\text { de medo, tristeza, angústia e } \\
\text { frustração, irritabilidade, insônia } \\
\text { e estresse, depressão, ansiedade. }\end{array}$ \\
\hline $\begin{array}{l}\text { Costa, Felipe de Almeida } \\
\text { et al.. (2020) - Brasil }\end{array}$ & $\begin{array}{l}\text { Idosos acima } \\
\text { de } 65 \text { anos de } \\
\text { idade }\end{array}$ & $\begin{array}{l}\text { Analisar e } \\
\text { investigar os } \\
\text { impactos do novo } \\
\text { coronavírus } \\
\text { causador da } \\
\text { pandemia de } \\
\text { COVID-19 no } \\
\text { metabolismo e } \\
\text { psicológico dos } \\
\text { idosos e possíveis } \\
\text { intervenções que } \\
\text { possam minimizar } \\
\text { esses impactos. }\end{array}$ & $\begin{array}{l}\text { Revisão qualitativa } \\
\text { exploratória da } \\
\text { literatura }\end{array}$ & $\begin{array}{l}\text { Evidenciou-se a necessidade de } \\
\text { ampliar a discussão sobre a } \\
\text { COVID-19, na perspectiva de } \\
\text { reduzir cada vez mais o número } \\
\text { de pacientes com infecções } \\
\text { pulmonares causadas pelo vírus, } \\
\text { assim como os efeitos } \\
\text { psicológicos que o isolamento } \\
\text { social tem propiciado nos } \\
\text { idosos. }\end{array}$ \\
\hline $\begin{array}{l}\text { Santos, Stephany da } \\
\text { Silva; Brandão, } \\
\text { Gisetti Corina Gomes; } \\
\text { Araújo, } \\
\text { Kleane Maria da } \\
\text { Fonseca Azevedo (2020) } \\
\text { - Brasil }\end{array}$ & $\begin{array}{l}\text { Idosos acima } \\
\text { de } 65 \text { anos de } \\
\text { idade }\end{array}$ & $\begin{array}{l}\text { Investigar } \\
\text { desafios } \\
\text { enfrentados } \\
\text { isolamento no } \\
\text { para a saúde mental } \\
\text { dos idosos durante } \\
\text { a pandemia do } \\
\text { COVID-19. }\end{array}$ & $\begin{array}{l}\text { Revisão Integrativa } \\
\text { da Literatura }\end{array}$ & $\begin{array}{l}\text { Reconhece-se a importância do } \\
\text { isolamento na profilaxia do } \\
\text { COVID-19, no entanto, } \\
\text { percebe-se que este pode } \\
\text { desencadear e/ou agravar } \\
\text { problemas psicológicos em } \\
\text { pessoas idosas. Alguns países } \\
\text { têm adotado estratégias para } \\
\text { assistir esta população em } \\
\text { situação de isolamento social. }\end{array}$ \\
\hline $\begin{array}{l}\text { Barros, Marilisa et al., } \\
(2020) / \\
\text { Brasil }\end{array}$ & 45.161 pessoas & $\begin{array}{lr}\text { Analisar } & \text { a } \\
\text { frequência } & \text { de } \\
\text { tristeza, ansiedade } \\
\text { e alterações do } \\
\text { sono durante } & \text { a } \\
\text { pandemia } & \text { de } \\
\text { COVID-19 } & \text { no } \\
\text { Brasil, } & \\
\text { identificando } & \text { os } \\
\text { segmentos } & \\
\text { demográficos } & \text { mais } \\
\text { afetados. }\end{array}$ & $\begin{array}{l}\text { Estudo transversal, } \\
\text { com o uso de } \\
\text { questionário } \\
\text { aplicado via web a } \\
\text { adultos e pessoas } \\
\text { idosas, rom } \\
\text { informações robre } \\
\begin{array}{lr}\text { condiçóes de vida, } \\
\text { saúde }\end{array} \\
\text { comportamento. }\end{array}$ & $\begin{array}{l}\text { A pandemia e isolamento social } \\
\text { impacta a saúde mental e a } \\
\text { qualidade do sono da população } \\
\text { no contexto brasileiro. As } \\
\text { pessoas com antecedentes de } \\
\text { depressão são as mais } \\
\text { vulneráveis. Entende-se como } \\
\text { necessária a aplicação e o } \\
\text { aprimoramento das estratégias } \\
\text { de atenção à saúde mental; } \\
\text { divulgação das medidas e } \\
\text { práticas de preservação da saúde } \\
\text { mental e da qualidade do sono } \\
\text { recomendadas pela OMS, } \\
\text { sociedades e associações de } \\
\text { profissionais da saúde mental. }\end{array}$ \\
\hline
\end{tabular}




\begin{tabular}{|c|c|c|c|c|}
\hline $\begin{array}{l}\text { Whitehead, B. R. } \\
\text { (2020)/Estados Unidos }\end{array}$ & $\begin{array}{l}874 \text { pessoas } \\
\text { residentes nos } \\
\text { Estados } \\
\text { Unidos. }\end{array}$ & $\begin{array}{lr}\text { Avaliou-se } & \text { a } \\
\text { associação } & \text { das } \\
\text { expectativas } & \text { de } \\
\text { pessoas idosas } & \text { em } \\
\text { relação a duração e } \\
\text { impacto } \quad \text { da } \\
\text { pandemia. }\end{array}$ & $\begin{array}{lr}\text { Os adultos } & \text { idosos } \\
\text { completaram } & \text { os } \\
\text { questionários sobre } \\
\text { estresse percebido } \\
(\text { OS), e afeto } \\
\text { negativo (NA) e as } \\
\text { expectativas em } \\
\text { relação à pandemia, } \\
\text { de modo online no } \\
\text { final de março de } \\
2020 \text {. }\end{array}$ & $\begin{array}{l}\text { Os resultados relatados } \\
\text { fornecem evidências de que as } \\
\text { expectativas diante da pandemia } \\
\text { influenciam no estresse e afeto } \\
\text { negativo dos idosos, trazendo } \\
\text { implicações para a saúde mental } \\
\text { dos mesmos. }\end{array}$ \\
\hline $\begin{array}{l}\text { Van Orden, K. A., et } \\
\text { al.(2020)/Estados Unidos }\end{array}$ & $\begin{array}{l}\text { Idosos acima } \\
\text { de } 65 \text { anos }\end{array}$ & $\begin{array}{l}\text { Analisar como os } \\
\text { médicos podem } \\
\text { ajudar os pacientes } \\
\text { mais velhos a } \\
\text { manter a saúde } \\
\text { social durante as } \\
\text { restrições de } \\
\text { distanciamento } \\
\text { social para } \\
\text { COVID-19. }\end{array}$ & 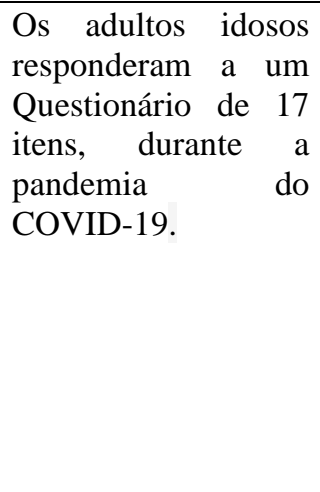 & $\begin{array}{l}\text { Estratégias } \\
\text { comportamentais } \begin{array}{r}\text { cognitivo- } \\
\text { foram }\end{array} \\
\text { utilizadas para promover a } \\
\text { conexão social para idosos } \\
\text { devido a restrições de } \\
\text { distanciamento social, incluindo } \\
\text { mudança de perspectivas, } \\
\text { enfrentamento da ansiedade, } \\
\text { prática de meditação } \\
\text { mindfulness e resolução de } \\
\text { problemas de novas maneiras } \\
\text { criativas de se conectar. }\end{array}$ \\
\hline $\begin{array}{l}\text { Pereira, Mara Dantas et } \\
\text { al.. (2020) - Brasil }\end{array}$ & $\begin{array}{l}\text { População em } \\
\text { geral, incluindo } \\
\text { idosos }\end{array}$ & \begin{tabular}{lr} 
Analisar & as \\
consequências & na \\
saúde & mental \\
advindas & do \\
período & de \\
isolamento & social \\
\multicolumn{2}{l}{ durante a pandemia } \\
\multicolumn{2}{l}{ de COVID-19 }
\end{tabular} & $\begin{array}{l}\text { Revisão integrativa } \\
\text { da literatura, } \\
\text { realizada a partir da } \\
\text { busca por } \\
\text { publicações } \\
\text { científicas. }\end{array}$ & 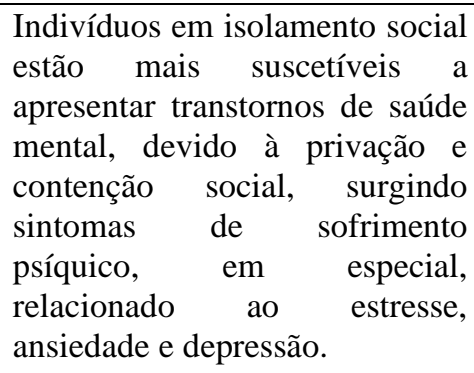 \\
\hline $\begin{array}{l}\text { Duarte, Michel de } \\
\text { Quadros et al.,(2020) - } \\
\text { Brasil }\end{array}$ & $\begin{array}{l}799 \text { residentes } \\
\text { no Rio Grande } \\
\text { do Sul }\end{array}$ & $\begin{array}{l}\text { Verificar os fatores } \\
\text { associados a } \\
\text { indicadores de } \\
\text { sintomas de } \\
\text { transtornos mentais } \\
\text { em residentes do } \\
\text { Rio Grande do Sul, } \\
\text { durante o período } \\
\text { inicial da política } \\
\text { de distanciamento } \\
\text { social decorrente } \\
\text { da pandemia da } \\
\text { COVID-19. }\end{array}$ & $\begin{array}{l}\text { Estudo transversal e } \\
\text { quantitativo, de } \\
\text { caráter exploratório } \\
\text { realizado com } \\
\text { indivíduos entre } 18 \text { e } \\
75 \text { anos, residentes } \\
\text { no Estado do Rio } \\
\text { Grande do Sul. }\end{array}$ & $\begin{array}{l}\text { Fazer parte do grupo de risco } \\
\text { para o novo coronavírus, ou } \\
\text { seja, ser gestante, ter acima de } \\
60 \text { anos ou doenças } \\
\text { preexistentes, como diabetes e } \\
\text { cardiopatias, significa um maior } \\
\text { risco de ter a doença em sua } \\
\text { forma agravada. Esses } \\
\text { participantes podem ter mais } \\
\text { chance de risco para transtornos } \\
\text { mentais menores. }\end{array}$ \\
\hline $\begin{array}{l}\text { Pavani, Fabiane } \\
\text { Machado et al.,(2020) - } \\
\text { Brasil }\end{array}$ & $\begin{array}{l}\text { Grupos } \\
\text { vulneráveis. }\end{array}$ & $\begin{array}{lr}\text { Identificar } & \text { as } \\
\text { repercussões } & \text { na } \\
\text { saúde mental } & \text { de } \\
\text { grupos } & \mathrm{e} \\
\text { populações } & \text { no } \\
\text { contexto } & \text { da } \\
\text { pandemia do novo } \\
\text { coronavírus. }\end{array}$ & Revisão narrativa. & $\begin{array}{l}\text { É fundamental traçar um plano } \\
\text { de cuidados, afim de melhorar } \\
\text { as funções psicológicas e } \\
\text { fisiológicas dos pacientes } \\
\text { adultos idosos, buscando } \\
\text { medidas que reduzam os efeitos } \\
\text { deletérios da pandemia, } \\
\text { diminuindo ou impedindo o } \\
\text { surgimento de desconforto } \\
\text { físico, social e emocional. }\end{array}$ \\
\hline
\end{tabular}




\begin{tabular}{|c|c|c|c|c|}
\hline $\begin{array}{l}\text { Nascimento Júnior, } \\
\text { Francisco Edmar do; } \\
\text { Tatmatsu, Daniely } \\
\text { Ildegardes Brito \& } \\
\text { Freitas, Rayanne } \\
\text { Gabrielle Torquato de. } \\
(2020) \text { - Brasil }\end{array}$ & $\begin{array}{l}\text { População } \\
\text { idosa acima de } \\
65 \text { anos }\end{array}$ & $\begin{array}{lr}\text { Analisar } & a \\
\text { influência } & \text { da } \\
\text { desconexão social e } \\
\text { da percepção do } \\
\text { isolamento social } \\
\text { sobre os quadros de } \\
\text { depressão } \\
\text { ansiedade. }\end{array}$ & Revisão de literatura & \begin{tabular}{lcr}
\multicolumn{3}{l}{ A variabilidade comportamental } \\
para acessar & reforçadores em \\
ambiente doméstico e a & e \\
aprendizagem & de & novo \\
repertório, & sobretudo r as \\
habilidades & digitais, para \\
continuar & \multicolumn{2}{c}{ interagindo } \\
socialmente e & discriminar a \\
qualidade e a & quantidade de \\
informações & necessárias, \\
tornaram-se & \multicolumn{2}{c}{ estratégias } \\
prioritárias para idosos no \\
cenário de COVID-19.
\end{tabular} \\
\hline $\begin{array}{l}\text { Ramirez-Ortiz, J. et al. } \\
\text { (2020) - Colômbia }\end{array}$ & $\begin{array}{l}\text { População em } \\
\text { geral }\end{array}$ & $\begin{array}{l}\text { Mostrar as } \\
\text { possíveis } \\
\text { consequências na } \\
\text { saúde mental da } \\
\text { população como } \\
\text { resultado do } \\
\text { isolamento } \\
\text { obrigatório devido } \\
\text { à pandemia de } \\
\text { COVID-19. }\end{array}$ & $\begin{array}{l}\text { Revisão Integrativa } \\
\text { da Literatura }\end{array}$ & $\begin{array}{l}\text { Essencial para implementar } \\
\text { estratégias destinadas a } \\
\text { antecipar esse impacto, nas } \\
\text { quais a prioridade tem sido dada } \\
\text { de acordo com a identificação } \\
\text { das populações vulneráveis ou } \\
\text { de alto risco. Dentro das } \\
\text { intervenções precoces incluem } \\
\text { primeiros socorros psicológicos } \\
\text { e grupos de apoio } \\
\text { multidisciplinar } \\
\text { complementada com terapias } \\
\text { cognitivo-comportamentais } \\
\text { voltadas para o trauma. }\end{array}$ \\
\hline $\begin{array}{l}\text { Silva, Marciele de Lima, } \\
\text { Viana, Suely Aragão } \\
\text { Azevedo \& Lima, } \\
\text { Patrícia Tavares de. } \\
\text { (2020) - Brasil. }\end{array}$ & Idosos & $\begin{array}{l}\text { Pesquisar } \\
\text { literatura estudos } \\
\text { que retratem os } \\
\text { impactos na saúde } \\
\text { mental dos idosos } \\
\text { durante o período } \\
\text { de isolamento } \\
\text { social como meio } \\
\text { de prevenção para a } \\
\text { disseminação da } \\
\text { doença COVID-19. }\end{array}$ & $\begin{array}{l}\text { Revisão de literatura } \\
\text { com abordagem } \\
\text { descritiva. }\end{array}$ & $\begin{array}{l}\text { O impacto na saúde mental dos } \\
\text { idosos devido à pandemia do } \\
\text { COVID-19 está relacionado ao } \\
\text { isolamento social, à diminuição } \\
\text { da dinâmica do dia a dia, ao } \\
\text { estresse gerado pelos cuidados } \\
\text { necessários para a prevenção e } \\
\text { até pelo excesso de } \\
\text { informações, portanto, este } \\
\text { estudo é de extrema relevância } \\
\text { para que as pessoas idosas } \\
\text { mantenham rotinas e tarefas } \\
\text { regulares sempre que possível e } \\
\text { crie novas num ambiente } \\
\text { diferente. }\end{array}$ \\
\hline
\end{tabular}

Fonte: Autores.

Dos estudos elencados nesta pesquisa, alguns foram realizados nos Estados Unidos ( $\mathrm{n}=2$ ), outros no Brasil ( $\mathrm{n}=9$ ) e na Colômbia $(n=1)$. Foram observadas as repercussões na saúde mental, as quais seriam produto de movimentos de exposição e proteção dos indivíduos ao longo do período de emergência em saúde pública alertada pela Organização Mundial de Saúde (OMS, 2020). Observaram-se também a manifestação de sintomas depressão, ansiedade, tipicamente concomitante, estresse e solidão, relacionados ao isolamento social.

\section{Discussão}

O objetivo deste estudo foi verificar o impacto causado pela pandemia COVID-19 na saúde mental das pessoas idosas. Apesar das diferenças culturais, observou-se que alguns sinais e sintomas afetaram a saúde mental de modo semelhante as populações de pessoas idosas no âmbito mundial, sendo importante discuti-los. 
É notória a importância de acatar medidas protetivas de distanciamento físico e social para controlar o avanço do vírus e minimizar seu impacto danoso na saúde da população idosa. Todavia, com o isolamento social pode haver a emergência ou o incremento de sentimentos de solidão e de outros sinais adversos à saúde, o que pode comprometer o funcionamento do sistema imunológico, aumentar as possibilidades de adultos idosos contraírem infecções por coronavírus, causar complicações e levá-los a óbito. Torna-se necessário, então, monitorar sentimentos de solidão, tristeza e sintomas depressivos, e buscar avaliar e intervir preventivamente, a fim de prevenir as consequências do distanciamento físico e social.

A presença de estados de angústia pode ser considerada uma resposta esperada diante de um evento da magnitude da pandemia COVID-19. Todavia o isolamento físico e social pode agregar efeitos de maior magnitude, nocivos a saúde mental dos indivíduos idosos e, da população geral, o que pode levar a um quadro psicopatológico. Compreender os fatores de influência dessa angústia pode reduzir o impacto geral e as consequências deste evento traumático pandêmico tanto no bemestar quanto na qualidade de vida das pessoas idosas (Whitehead, 2020).

Em tempos de distanciamento físico e social, as medidas protetivas contra o vírus, decretadas pelos órgãos públicos, podem alterar o ritmo da vida das pessoas idosas, tornando-se restritivas para a consolidação de atividades rotineiras, as quais lhes proporcionavam segurança e amparo. Sensações de medo, solidão, podem emergir e afetar negativamente a saúde mental. No caso de indivíduos idosos que não convivem com suas famílias, é importante que seja criado um plano de conexão, que os ajude a enfrentar a solidão causada pelo distanciamento. Essas conexões permitem muitos tipos de relações com outras pessoas, com animais e com a natureza (Van Orden et al., 2020).

Os estudos encontrados a nível nacional apontaram que há particularidades no modo como a pandemia impacta as pessoas idosas, considerando seu nível de saúde anterior a pandemia, mas de maneira geral, estão relacionados a sentimentos de medo, tristeza, ansiedade, angústia, irritabilidade, insônia e estresse, depressão (Garcia \& Machado, 2020). O impacto local irá variar e evoluir dependendo de fatores específicos, incluindo a incidência de COVID-19, as taxas de mortalidade associadas, a disponibilidade de recursos e as mudanças sociais implementadas para controlar a pandemia.

Pôde-se observar que no Brasil, sentimentos de tristeza/depressão, nervosismo/ansiedade e problemas de sono atingiram o maior índice em março 2020 em adultos que aderiram o isolamento social, sendo que no mês de abril observou-se um declínio desta adesão, o que demonstrou que parte da população deixou de atender integralmente às recomendações de distanciamento. Esse fenômeno pode ter sido reforçado pela propagação de informações ambíguas ou falsas em relação à COVID-19 (Barros et al., 2020).

A experiência de estresse de adultos idosos no início da pandemia alertou para o impacto de longo alcance e implicações desconhecidas de prazo indeterminado. Os resultados relatados fornecem evidências de que as expectativas diante da pandemia influenciam no estresse e afeto negativo de indivíduos idosos, incidindo em alterações na saúde mental (Whitehead, 2020). Em estudo sobre a frequência de tristeza, ansiedade e alterações do sono durante a pandemia de COVID-19 no Brasil que, durante o período da pandemia e de distanciamento social de abril e maio de 2020, houve menos relatos de pessoas idosas que se sentiam tristes ou deprimidos, nervosos ou ansiosos, esses sentimentos atingiram mais os adultos jovens (Barros et al., 2020).

Para Costa et al. (2020), há necessidade de ampliar a discussão sobre a COVID-19, na perspectiva de reduzir cada vez mais o número de pacientes com infecções pulmonares causadas pelo vírus, assim como os efeitos psicológicos que o isolamento físico e social tem propiciado em pessoas idosas. Segundo Souza (2020), o isolamento social tem caráter de prevenção para as pessoas idosas, e necessariamente não precisa ser acompanhado de isolamento cognitivo ou funcional. Com o intuito de diminuir e evitar possíveis estados de ansiedade uma intervenção que pode ser realizada é a inserção dos idosos no mundo digital para que estes se sintam menos isolados dos amigos, familiares e segregados do mundo. 
A inclusão digital promove melhoria da qualidade de vida das pessoas idosas, permite o acesso a inúmeros serviços, como operações bancárias e compras on-line, facilita a obtenção de informações e amplia a sociabilidade (Nascimento Júnior et al., 2020). Entretanto, o aprendizado para o acesso às Tecnologias da Informação e da Comunicação (TIC), o letramento e a inclusão digital, podem ser um desafio para muitas pessoas idosas. Com o objetivo de auxiliar a vencer alguns desses entraves e assistir à população idosa durante a pandemia, vídeos tutoriais de como instalar aplicativos e utilizar canais digitais foram disponibilizados por diversas empresas. Adicionalmente, outros materiais como guia de orientação também foram disponibilizados na modalidade on-line (Scortegagna, Pasian, Lima, 2021).

Nessa perspectiva, a pandemia abre possibilidades para que se possa criar alternativas inovadoras para suprir as necessidades humanas de cuidado familiar e convívio social. Motiva familiares (filhos, netos, sobrinhos, etc.) a auxiliarem nos ensinamentos das pessoas idosas quanto ao uso de dispositivos tecnológicos como computadores, smartphones, tablets e outros, de modo a aproximar relações familiares e entre amigos, diminuir sintomas adversos como a ansiedade, assegurar atividades diárias e manter rituais religiosos ou celebrar datas como aniversários, fomentar a autonomia, e preservar a autoestima. Manter contato familiar telefônico, sem contato visual, também pode permitir o acesso a reforçadores da saúde mental, ainda que de menor intensidade (Nascimento Júnior et al., 2020).

Desse horizonte, importa as pessoas idosas não se sentirem desamparadas pela redução das visitas ou contatos. Importa, ainda, que os cuidadores mantenham uma rotina com adequações necessárias, voltando-se principalmente para a qualidade de vida - física e psicológica dos seus familiares idosos. O confinamento imposto pela COVID-19, tem posto à prova a capacidade humana de dar sentido ao sofrimento e desafiado tanto os indivíduos quanto a sociedade, no Brasil e em todo o planeta, a promoverem formas de coesão que minimizem o impacto de experiências-limite na vida mental (Lima, 2020).

O isolamento social entre os idosos traz grande preocupação aos órgãos de saúde pública visto que, aumenta o risco de aparecer problemas cardiovasculares, autoimunes, neurocognitivos e de saúde mental (Armitage \& Nellums, 2020). Indivíduos idosos com doenças mentais, são mais propensos a desenvolverem depressão e sintomas de ansiedade e correm risco maior de recaídas devido a esse cenário emergente, pois dependem de outros para realizar suas atividades da vida diária (Mehra et al., 2020).

De forma geral, os resultados relatados sintetizam um primeiro passo para a compreensão da vivência de estresse em pessoas idosas no início da pandemia, um estressor singular e seu abalo de longa abrangência e implicações desconhecidas em longo prazo. Se os adultos idosos estiverem preparados para uma comoção mais longa e permanente sabendo do risco de sofrimento, podem adotar medidas pró ativas e recursos de enfrentamento eficazes para reduzir o estresse antes que ele traga danos a saúde física e mental.

As consequências da pandemia mostram sintomas de estresse em grande parte da população afetada. As incertezas provocadas pelo COVID-19, os riscos de contaminação e o isolamento físico e social podem agravar ou gerar problemas mentais. Portanto, algumas intervenções são necessárias para superar este período da melhor maneira possível, tais como: demonstrar empatia; reduzir o estigma de quem foi infectado; informar as pessoas com notícias verídicas; prover cuidados com crianças, profissionais da saúde que se encontram na linha de frente ao combate ao vírus e outros grupos de riscos como as pessoas idosas (OMS, 2020). Idosos, especialmente os que se encontram em isolamento social e que apresentam algum quadro cognitivo como demência podem se tornar mais ansiosos, agitados, agressivos e distanciados durante a quarentena (Silva, Viana \& Lima, 2020). Oferecer a eles apoio emocional por meio dos familiares, amigos ou de agentes de saúde, partilhar fatos simples com informações claras, concisas, a respeito da redução de riscos e infecções, repetir as informações sempre que necessário e utilizar outros meios de comunicação além do verbal, como a escrita, pinturas e figuras, podem ser recursos eficazes (OMS, 2020). 
As quarentenas tendem a despertar e agravar sentimentos como solidão, estresse, ansiedade, tristeza, depressão, entre outros. Para prover assistência orientam-se medidas de autoajuda, como assistir filmes, ler um livro, praticar atividades físicas, ter apoio de um profissional da área da saúde para esclarecer, diagnosticar transtornos mentais e oferecer um tratamento adequado. Geralmente, pessoas idosas temem por si e por seus entes queridos, apresentam alteração no sono, no apetite, o que pode agravar problemas crônicos de saúde como diabetes, hipertensão e doenças cardiovasculares (Silva, Viana \& Lima, 2020). Ser idoso ou ter doenças preexistentes, como diabetes e cardiopatias, significa um maior risco de ter a doença COVID19 em sua forma agravada e ter mais chance de risco para transtornos mentais menores (Duarte et al.,, 2020).

Em razão da pandemia o acesso aos cuidados de saúde mental de qualidade e acessíveis no mundo ficou ainda mais limitado, pois foram interrompidos pela pandemia. Nos últimos meses, a OMS emitiu orientações e aconselhamentos sobre saúde mental para todos os profissionais que trabalham na linha de frente de combate ao COVID-19 e pessoas de todas as idades cujas vidas mudaram consideravelmente como resultado da pandemia. Com a interrupção dos serviços de saúde presenciais, os países buscam maneiras inovadoras de fornecer cuidados de saúde mental e surgiram iniciativas para fortalecer o apoio psicossocial. No entanto, devido à escala do problema, a maioria das necessidades de saúde mental permanece sem solução. A resposta é dificultada por problemas crônicos de sub financiamento em promoção, prevenção e atenção à saúde mental, problema que ocorre há muitos anos, antes da COVID-19 (OPAS/BRASIL, 2020).

Conforme Nascimento Júnior, Tatmatsu \& Freitas (2020), a variabilidade comportamental para acessar reforçadores em ambientes domésticos e a aprendizagem de novo repertório, sobretudo as habilidades digitais, para continuar interagindo socialmente e discriminar a qualidade e a quantidade de informações necessárias, tornaram-se estratégias prioritárias para pessoas idosas no cenário de COVID-19. Mas, vale ressaltar que nem todos os indivíduos idosos se adaptam ao uso de dispositivos eletrônicos. Tal fato pôde ser observado pelo aumento de sedentarismo como resultado da resistência de alguns adultos idosos em usar ferramentas online para atividades físicas.

A campanha do Dia Mundial da Saúde Mental oferece oportunidades, principalmente virtuais devido à contínua pandemia, para que todos façam algo que afirme a vida: como indivíduos, tomar ações concretas em apoio à própria saúde mental e apoiar amigos e familiares que estão lutando; aos empregadores, tomar medidas para implementar programas de bemestar dos funcionários; aos governos, comprometer-se a estabelecer ou ampliar os serviços de saúde mental; e aos jornalistas, para explicar o que mais pode e deve ser feito para tornar a assistência à saúde mental uma realidade para todos (OPAS/BRASIL, 2020).

Estudos que visam analisar os impactos causados pela pandemia do COVID-19 na saúde mental da população e quais os meios possíveis para o seu enfrentamento estão sendo realizados de forma a contribuir para uma ampla avaliação do contexto em saúde mental, especialmente a Psicologia da Saúde, e áreas afins. Programas de cuidado psicológico para lidar com as diferentes situações que ocorrem ao longo da pandemia, foram predominantemente relacionados ao atendimento online, focalizando a compreensão do ajustamento psicológico frente às adversidades, especialmente as com alto potencial de severidade e alcance como a COVID-19 (Faro et al., 2020).

No Brasil, alguns projetos de pesquisa inovadores que visam a assistência on-line de populações mais vulneráveis ao COVID-19 foram implementados. Entre estes destaca-se o projeto intitulado "Coletivos On-line em Saúde Mental: Ação Transdisciplinar para Grupos Vulneráveis a Covid-19”, aprovado pelo edital FAPERGS 06/2020 - Ciência e Tecnologia no Combate à COVID-19 (https://coletivosaudemental.com.br/pesquisa/). Como objetivo geral, este estudo empírico busca avaliar e ofertar estratégias de suporte on-line, em saúde mental e condições gerais de saúde, a pessoas idosas e aos profissionais da saúde. Trata-se de uma investigação multicêntrica em desenvolvimento por três centros de excelência no ensino-pesquisaextensão universitária: a Universidade de Passo Fundo (Programa de Pós-Graduação em Envelhecimento Humano), no Rio 
Grande do Sul; a Universidade de São Paulo (Programa de Pós-Graduação em Psicologia), em Ribeirão Preto, São Paulo; e a Universidade de Brasília (Programa de Pós-Graduação em Psicologia Clínica e Cultura), Distrito Federal.

Algumas manifestações ansiosas e depressivas em pessoas em isolamento social devido a pandemia, podem ser evidenciadas por reações ao estresse. Percebe-se que a perda de controle nessas circunstâncias é recorrente, pois em muitos casos o contexto impossibilita que o indivíduo saiba o tempo determinado em que a crise será solucionada. Estes sentimentos de incertezas, como também os limites impostos pelas medidas preventivas de isolamento social, a iminência de alterações nos planos futuros de modo drástico, além da separação brusca do ambiente social ou familiar do indivíduo, se tornam catalisadores constantes para o surgimento de sintomas de ansiedade e de quadros depressivos (Pereira et al.,, 2020). Nesse contexto, reconhece-se a importância do isolamento na profilaxia do COVID-19, no entanto, percebe-se que este pode desencadear e/ou agravar distúrbios psicológicos em indivíduos idosos que necessitam de estratégias para o enfrentamento deste isolamento que mudou a frequência da comunicação com amigos próximos e familiares, daí a importância do uso de dispositivos de on-line.

É fundamental traçar um plano de cuidados, afim de melhorar as funções psicológicas e fisiológicas dos pacientes idosos, buscando medidas que reduzam os efeitos deletérios da pandemia, diminuindo ou impedindo o surgimento de desconforto físico, social e emocional. Para amenizar o impacto negativo do distanciamento físico e social, prática de meditação mindfulness e resolução de problemas de novas maneiras criativas de se conectar mostrou-se um importante mecanismo em que, por meio de vídeo-chamadas, possibilitou o contato, amenizando os impactos do isolamento (Van Orden et al., 2020).

Ramirez-Ortiz et al., (2020), consideram essencial implementar estratégias destinadas a antecipar esse impacto, nas quais a prioridade tem sido dada de acordo com a identificação das populações vulneráveis ou de alto risco. As intervenções precoces incluem primeiros socorros psicológicos e grupos de apoio multidisciplinar e complementada com terapias cognitivocomportamentais voltadas para o trauma. Além disso, alguns pesquisadores salientam a necessidade de se traçar planos de cuidados, a fim de melhorar as funções psicológicas e fisiológicas dos pacientes idosos, e buscar medidas que reduzam os efeitos deletérios da pandemia para diminuir ou impedir o surgimento de desconforto físico, social e emocional (Pavani et al., 2020).

Finalmente, diante da pandemia, pesquisadores buscam uma vacina, o que gera esperanças principalmente para adultos idosos inseridos em grupos de risco. A Fundação Oswaldo Cruz (FIOCRUZ) tem desempenhado papel central no enfrentamento da pandemia de COVID-19 no Brasil e na produção de vacinas para COVID-19. A principal aposta da Fiocruz foi um acordo com a biofarmacêutica AstraZeneca para produzir, no Brasil, a vacina contra o novo coronavírus desenvolvida pela Universidade de Oxford. Na primeira fase estão previstas vacinações em trabalhadores da saúde, pessoas com mais de 75 anos, indivíduos acima dos 60 anos que vivem em instituições de longa permanência e hospitais psiquiátricos (FIOCRUZ, 2020). A vacinação da população gera esperanças de que a vida social seja retomada, que o país atenda as consequências de pandemia na saúde e retome seu crescimento econômico.

\section{Considerações Finais}

Ao investigar os efeitos da COVID-19 na saúde mental de pessoas idosas, este estudo traz compreensões sobre o arcabouço que envolve o atual momento pandêmico que o mundo vive. As recomendações de isolamento e de distanciamento social impõem limitações de contato interpessoal presencial às populações idosas com familiares e amigos, suscitando ou agravando sentimentos de solidão, ansiedade, sintomas depressivos, dentre outros. Nesse contexto, o uso de TIC e seus 
benefícios são destacados como dispositivos para resgatar a conexão social e familiar, e minimizar os impactos do isolamento na saúde mental.

Vale ressaltar que, os estudos analisados conjuntamente trouxeram diretrizes para beneficiar o cuidado com o sofrimento da população, com suas incertezas quanto ao futuro e as inúmeras perdas e lutos sofridos, buscando reduzir danos a saúde mental e prevenir a emergência de transtornos psiquiátricos, principalmente nos adultos idosos. Muito embora a pandemia possa impor desafios inimagináveis, ela traz inquietações quanto a necessidade de aprimoramento de métodos e técnicas capazes de auxiliarem no enfrentamento de adversidades que ainda estão por vir, no período pós pandêmico. Por fim, a escassez de estudos relacionados a esta temática impulsiona a necessidade de novas pesquisas que contemplem os efeitos da pandemia em diferentes períodos no Brasil, e a eficácia das intervenções on-line dirigidas especialmente a população de adultos idosos.

\section{Referências}

Brooks, S. K., Webster, R. K., Smith, L. E., Woodland, L., Wessely, S., Greenberg, N., \& Rubin, G. J. (2020). The psychological impact of quarantine and how to reduce it: rapid review of the evidence. The Lancet, 395(10227), 912-920.

Brown, E. E., Kumar, S., Rajji, T. K., Pollock, B. G. \& Mulsant, B. H. (2020). Anticipating and Mitigating the Impact of the COVID-19 Pandemic on Alzheimer's Disease and Related Dementias. Am J Geriatr Psychiatry. 18 abr.

Costa, F. A., Silva, A. S., Oliveira, C. B. S., Costa, L. C. S., Paixão, M. E. S., Celestino, M. N. S., Araújo, M. C., Azevedo, S. M. A., Silva, C. R. C. \& Santos, I. L. V. L. (2020) COVID-19: seus impactos clínicos e psicológicos na população idosa. Brazilian Journal Development. V. 6, n. 7.

Duarte, M. Q., Santo, M. A. S., Lima, C. P., Giordani, J. P. \& Trentini, C. M. (2020). COVID-19 e os impactos na saúde mental: uma amostra do Rio Grande do Sul, Brasil. Ciência \& Saúde Coletiva, 25(9):3401-3411.

Faro, A., Bahiano, M. A., Nakano, T. C., Reis, C., Silva, B. F. P. \& Vitti, L. S. (2020). COVID-19 e saúde mental: A emergência do cuidado. SciElo, Estudos de Psicologia, Campinas, vol.37, jun. 2020.

Fundação Oswaldo Cruz - Fiocruz. (2020). Vacinas contra Covid-19. Dez. Rio de Janeiro.

Garcia, E. B. \& Machado, L. M. (2020). Saúde mental e emocional das pessoas idosas em tempos de pandemia. Congresso Internacional Interfaces da Psicologia aproximando distâncias. 11, 12 e 13 de Novembro.

Hossain, M. M., Sultana, A. \& Purohit, N.. (2020). Mental health outcomes of quarantine and isolation for infection prevention: A systematic umbrella review of the global evidence. PsyArXiv, 1-27.

Lima, R. C. (2020). Distanciamento e isolamento sociais pela Covid-19 no Brasil: impactos na saúde mental. Physis: Revista de Saúde Coletiva, Rio de Janeiro, v. 30(2), e300214.

Ministério da Saúde - MS. (2020). Sobre a doença: como se proteger. Brasil.

Moher, D., Liberati, A., Tetzlaff, J. \& Altaman, D. G. (2009). Preferred Reporting Items for Systematic Reviews and Meta-Analyses: The PRISMA Statement. Plos Medicine. 21 jul.

Nascimento Júnior, F. E., Tatmatsu, D. I. B. \& Freitas, R.G. T. (2020. Ansiedade em idosos em tempos de isolamento social no Brasil (COVID-19). Revista Brasileira de análise do comportamento / Brazilian Journal of Behavior Analysis, Vol. 16, N.1, 50-56.

Organização Mundial da Saúde - OMS. (2020). Publicações da OMS.

Organização Pan-Americana da Saúde -OPAS. (2020) Folha informativa COVID-19- Brasil. OPAS. 14 de ago.

Pavani, F. M., Silva, A. B., Olschowsky, A., Wetzel, C., Nunes, C. K. \& Souza, L. B. (2020). Covid-19 e as repercussões na saúde mental: estudo de revisão narrativa de literatura. Rev Gaúcha Enferm. 2021;42(esp):e20200188.

Pereira, M., Oliveira, L. C., Costa, C. F. Y., Bezerra, C. M. O., Pereira, M. D., Santos, C. K. A. \& Dantas, E. H. M. (2020). A pandemia de COVID-19, o isolamento social, consequências na saúde mental e estratégias de enfrentamento: uma revisão integrativa. Revista Research, Society and Development; maio.

Ramirez- Ortiz, J., Castro-Quintero, D., Lerma-Córdoba, C., Yela-Ceballos, F., \& Escobar-Córdoba, F. (2020). Consecuencias de la pandemia COVID-19 en la Salud Mental asociadas al aislamiento social. SciELO Preprints, 1, 1-21.

Santos, S. S., Brandão, G. C. G., Araújo, K. M. F. A. (2020). Social isolation: a look health elderly mental during the COVID-19 pandemic. Research, Society and Development, 9(7): 1-15.

Scortegagna, S.A., Pasian, S.R., Lima, M.A. (2021). Coletiv@s on-line em saúde mental: ação transdisciplinar para grupos vulneráveis à covid 19: Cartilha informativa e guia de saúde mental. UPF Editora. 
Research, Society and Development, v. 10, n. 7, e50210716947, 2021

(CC BY 4.0) | ISSN 2525-3409 | DOI: http://dx.doi.org/10.33448/rsd-v10i7.16947

Silva, M. L., Viana, S. A. A. \& Lima, P. T.. (2020).. O impacto na saúde mental do idoso durante o período de isolamento social em virtude da disseminação da doença covid-19: uma revisão literária. Revista Diálogos em Saúde. Vol. 3, n 1, jan/junho.

Souza, J. H. A. (2020). Isolamento social versus qualidade de vida dos idosos: um olhar multiprofissional frente à pandemia do Covid-19. Pubsaúde, 3 , a035.

Van Orden, K. A., Bower, E., Lutz, J., Silva, C. Gallegos, A. M., Podgorsky, C. A., Santos, E. J. \& Conwell, Y. (2020). Strategies to Promote Social Connections Among Older Adults During Social Distancing; Restrictions. Am J Geriatr Psychiatry. 18 mai.

Wang, H., Li, T., Barbarino, P., Gauthier, S., Brodaty, H., Molinuevo, J. L., Xie, H., Sun, Y., Yu, E., Tang, Y., Weidner, W. \& Yu, X. (2020). Dementia care during COVID-19. Lancet (London, England), 395(10231): 1190.

Whitehead, B. R. (2020). COVID-19 as a Stressor: Pandemic Expectations, Perceived Stress, and Negative Affect in Older Adults. J Gerontol B Psychol Sci Soc Sci. 04 set.

World Health Organization - WHO. (2020a). Mental health and psychosocial considerations during the COVID-19 outbreak. 